\title{
HIGHER-ORDER NON-LINEAR ANALYSIS OF STEEL STRUCTURES PART I : ELASTIC SECOND-ORDER FORMULATION
}

\author{
C.K. Iu ${ }^{*}$ and M.A. Bradford \\ Centre for Infrastructure Engineering and Safety \\ School of Civil and Environmental Engineering \\ The University of New South Wales, UNSW Sydney, NSW 2052, Australia \\ *(Corresponding author: E-mail: iu.jerryu@gmail.com)
}

Received: 31 May 2011; Revised: 10 October 2011; Accepted: 14 November 2011

\begin{abstract}
This paper presents a higher-order beam-column formulation that can capture the geometrically non-linear behaviour of steel framed structures which contain a multiplicity of slender members. Despite advances in computational structural frame software, analyses of large frames can still be problematic from a numerical standpoint, with efficacious and reliable convergence not always being ensured. To this end, the intent of this paper is to fulfil a need for versatile, reliable and efficient non-linear analysis of general steel framed structures with a large number of members suitable for engineering practice. Following a comprehensive review of numerical frame analysis techniques, a fourth-order element is derived, in which the crucial member bowing effect involved in the equilibrium equation is captured, and implemented in an updated Lagrangian formulation. Because of this, it is able to predict flexural buckling, snap-through buckling and the large displacement post-buckling behaviour of typical structures whose responses have been reported by independent researchers. The present approach with its efficacious and reliable convergence is shown in comparison studies to be applicable to selected applications which are prone to several forms of geometric non-linearity.
\end{abstract}

Keywords: Beam-column, Frames, Geometric non-linearity, Higher-order element, Steel, Updated lagrangian

\section{INTRODUCTION}

Displacement-based finite element techniques have been the most favoured for frame structures, mainly because of their accuracy, versatility, fully-established mathematical (variational) basis, and their suitability for computer implementation. The finite element method is based on the general principle that equilibrium of the structure under applied loading is achieved at displacements which correspond to the potential energy of the structure being stationary. In the description of the kinematic deformations of framed structures is favourably based on an updated Lagrangian system. Wen and Rahimzadeh [1] and Meek and Tan [2] employed a cubic displacement function to develop their displacement-based finite element method, while So and Chan [3] developed a quartic finite element, whose additional freedom in the displacement function was evaluated using the mid-span deflection of an element; a quintic element (PEP) was presented by Chan and Zhou [4] on the basis of force equilibrium at mid-span to quantify the large displacement behaviour of framed structures. Based on this, Chan and Zhou [5] incorporated initial imperfections into the element formulation, for which a single element can capture buckling of a member under axial compression alone. More recently, Gu and Chan [6] presented refined element including flexural and torsional buckling with finite rotations. Liew et al. [7] proposed a second-order analysis which allows for the geometric non-linear behaviour using one element. Their formulation makes use of stability functions for the transverse displacements, and it also considers the elastic coupling of axial, flexural and torsional deformations. Later, Liew et al. [8, 9] developed their numerical non-linear analysis of a structure subjected to fire and explosion. Similarly, Iu et al. [10,11] presented the non-linear fire analysis of steel structure including heating and cooling behaviour. 
Contemporary steel framed structures invariably contain a multiplicity of slender members, which makes them prone to instability effects so that large displacements and various kinds of buckling can occur because of geometrical non-linearities. For large framed structures containing many members, accurate solutions with computational expediency for the member forces and the structural deformations are needed for engineering practice. Despite significant advances in computational treatments of geometric and material non-linearities in structural frames, the non-linear analysis of slender framed structures with many members is still problematic, with accurate and efficient solutions not always being achievable for large-scale structures. In order to facilitate such a solution, a higher-order displacement-based finite element procedure is proposed in the present paper, based on a fourth-order element. Modern performance-based design paradigms for extreme loading events such as fire, blast and earthquake loads require accurate and efficient techniques, such as that developed in this paper. It is shown that this higher-order element, derived with reference to an incremental updated Lagrangian formulation, is able to provide efficient solutions for structures experiencing significant geometric non-linearity. It also provides the basis for including material non-linearity, which is introduced in the companion paper.

\section{FINITE ELEMENT FORMULATION}

The deformations along the member are taken as $\mathbf{u}=\{u, v, w, \phi\}^{\mathrm{T}}$, which comprise of the deformations $u$ in the $x$ direction, $v$ in the $y$ direction, $w$ in the $z$ direction and the twist $\phi$ about the $x$-axis. The member is idealised in a finite element formulation from the standpoint of having only one element, with the displacement functions chosen being required to satisfy the kinematic condition containing the $\mathrm{P}-\delta$ induced second-order moment as depicted in Figure 1 . The nodal displacement functions of the element are based on nodal displacements in a co-rotational coordinate, so that the dependent variables for the transverse displacements $v$ and $w$ are replaced by the nodal rotations $\theta_{z}$ and $\theta_{y}$ about the $z$ and $y$ axes respectively. These rotations are the dependent variables which define the transverse displacements in the element stiffness formulation which follows.

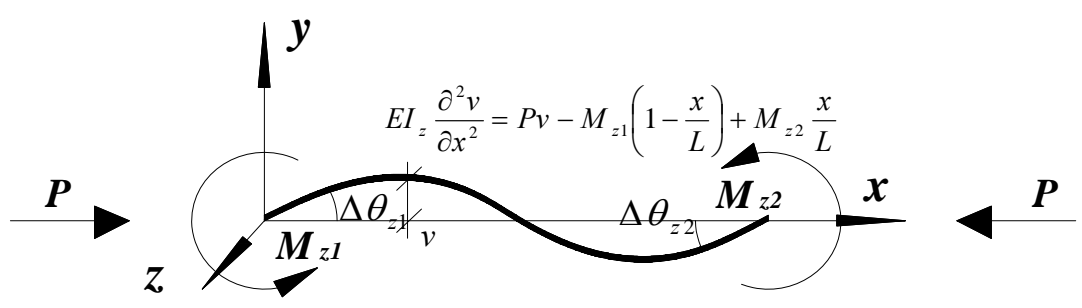

a) Equilibrium of a beam-column element about z-axis

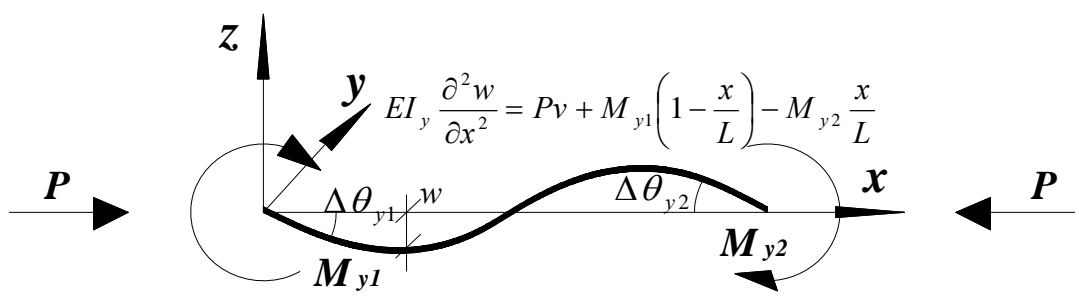

b) Equilibrium of a beam-column element about $y$-axis

Figure 1. Equilibrium Conditions for Deflected Beam-column Element 
Linear functions are assumed for the axial deformation and twist, so that

$u=(1-\xi) u_{1}+\xi u_{2}$

$\phi=(1-\xi) \theta_{x 1}+\xi \theta_{x 2}$

in which $u=u_{1}$ at $x=0, u=u_{2}$ at $x=L$ are the axial nodal deformations, $\phi=\theta_{x 1}$ at $x=0, \phi=\theta_{x 2}$ at $x=L$ are the twist nodal deformations, and where

$\xi=\frac{x}{L}$.

This paper uses fourth-order displacement functions to simulate member bowing behaviour; this necessitates the use of an additional equilibrium condition to tackle an additional unknown coefficient in the fourth-order term which constitutes a secondary or statical boundary condition. This moment equilibrium equation includes the second-order moment due to the member bowing effect and is introduced in the proposed displacement function, and subsequently its stiffness formulation. For the transverse displacement $v$ in the $y$ direction, the primary boundary conditions are

$v=0 \quad$ and $\quad \frac{\partial v}{\partial x}=\theta_{z 1} \quad$ at $x=0$

and

$v=0 \quad$ and $\quad \frac{\partial v}{\partial x}=\theta_{z 2} \quad$ at $x=L$,

while the equation of bending given by

$E I_{z} \frac{\partial^{2} v}{\partial x^{2}}=P v-M_{z 1}(1-\xi)+M_{z 2} \xi$

produces

$E I_{z} \frac{\partial^{2} v}{\partial x^{2}}=P v+\frac{M_{z 2}-M_{z 1}}{2} \quad$ at $\xi=1 / 2$

and which leads to the deformation

$$
\begin{aligned}
v= & {\left[\xi-\frac{4(24+q) \xi^{2}}{48+q}+\frac{(48+5 q) \xi^{3}}{48+q}-\frac{2 q \xi^{4}}{48+q}\right] L \theta_{z 1} } \\
& +\left[\frac{-(48-q) \xi^{2}}{48+q}+\frac{3(16-q) \xi^{3}}{48+q}+\frac{2 q \xi^{4}}{48+q}\right] L \theta_{z 2}
\end{aligned}
$$

in which

$q=\frac{P L^{2}}{E I}$ 
The transverse displacement $w$ in the $z$ direction is derived in a similar fashion. It should be noted that when the stability parameter $q=0$, the transverse displacements reduce to the conventional cubic deformations.

In order to verify the use of the displacement functions given in Eqs. 8, the displacements of the propped cantilever shown in Figure 2 with a tip rotation of 1 radian have been determined based on stability functions, as well as using a third-order polynomial, the present quartic representation, and using a fifth-order polynomial. In the range $0 \leq q \leq \pi^{2}$ (the limit $q=\pi^{2}$ representing the Euler load), the present representation is in good agreement with the exact solution. However, it can be seen that the quartic does produce underestimates of the deformations at very high axial compressive forces $\left(q=2 \pi^{2}\right)$, but this level of axial force is argued to be within the range of inelastic buckling for which elastic stability considerations are not significant.

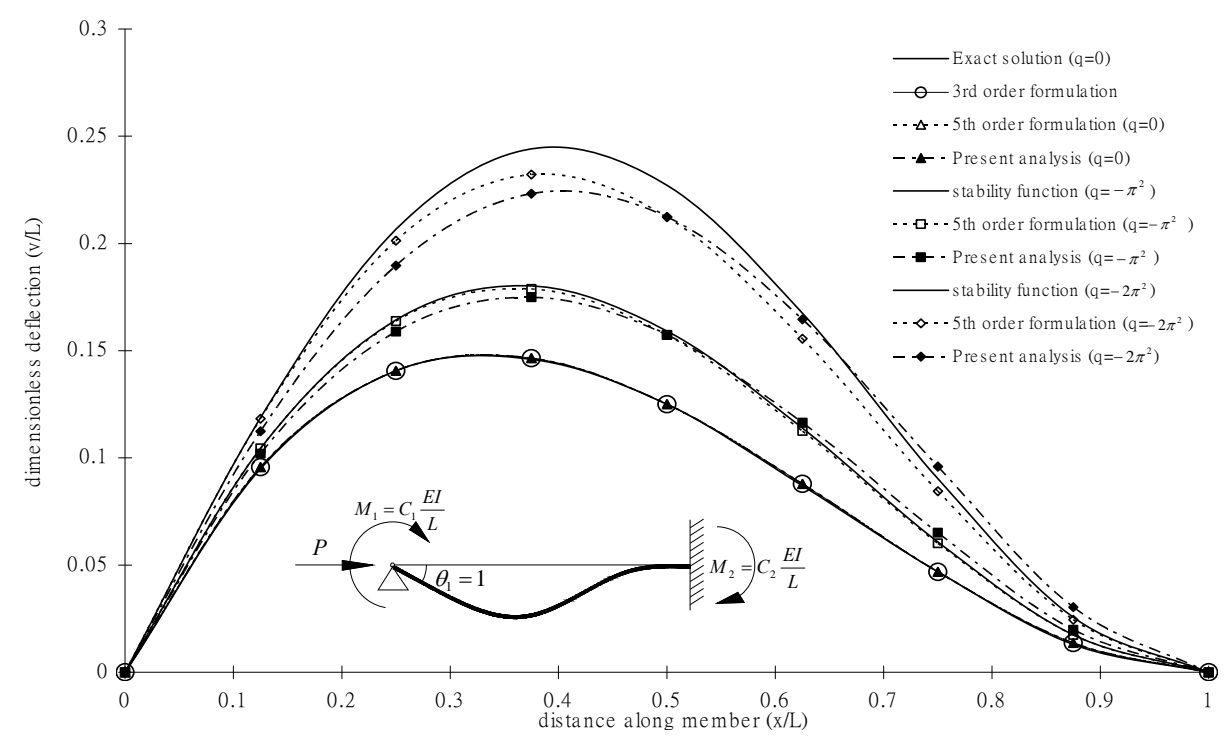

(a) Unit Rotation at Left Hand Support

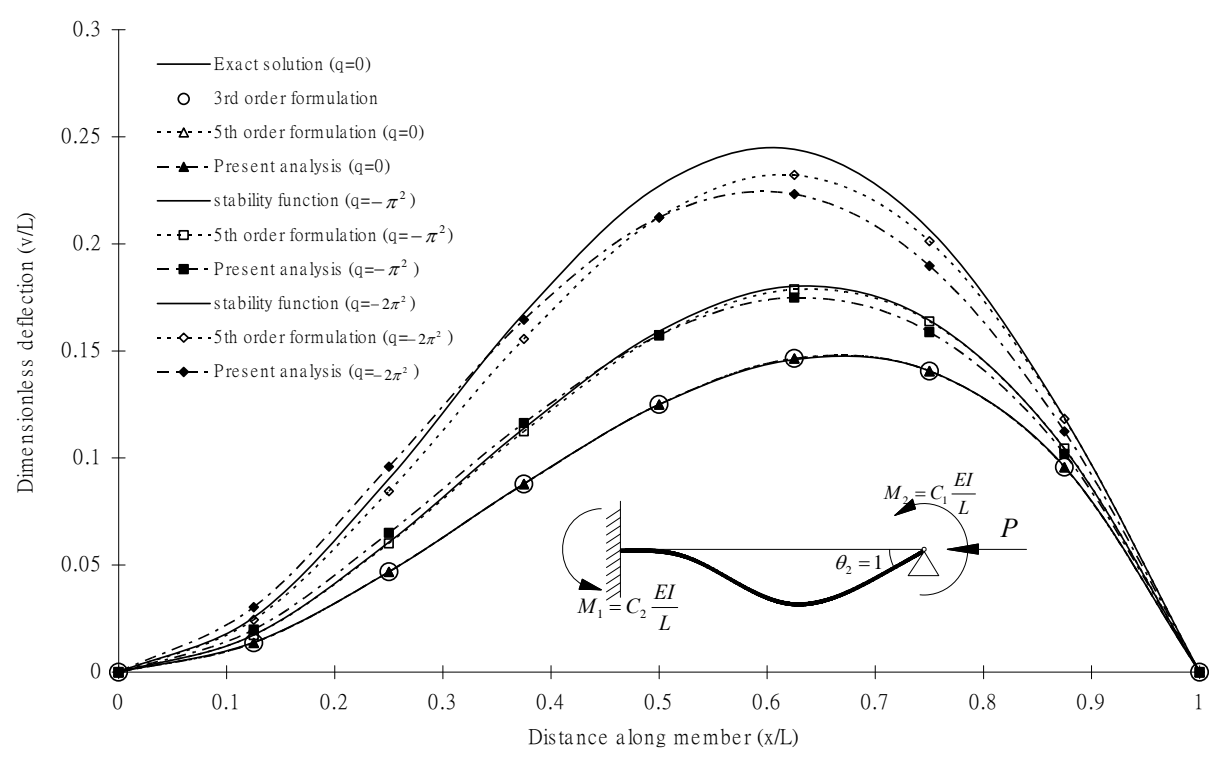

(b) Unit Rotation at Right Hand Support

Figure 2. Deflections of a Propped Cantilever 


\section{STIFFNESS FORMULATION FOR $4^{\text {TH }}$ ORDER BEAM-COLUMN ELEMENT}

The internal strain energy $U$ caused by the axial strain $\varepsilon_{x}$ and twist strain $\gamma_{x}$ along the beam-column continuum can be accumulated by integration of $\delta U_{A}=E \varepsilon_{x} \delta \varepsilon_{x}$ and of $\delta U_{T}=G \gamma_{x} \delta \gamma_{x}$ over the domain of length, which can be expressed in terms of $u, v, w$ and $\phi$ from an appropriate expansion of Green’s strain tensor as

$$
U=\int_{V o l}\left(\int_{\varepsilon_{x}} E \varepsilon_{x} \mathrm{~d} \varepsilon_{x}+\int_{\gamma_{x}} G \gamma_{x} \mathrm{~d} \gamma_{x}\right) \mathrm{d} V o l=\frac{1}{2} \int_{V o l}\left(E \varepsilon_{x}^{2}+G \gamma_{x}^{2}\right) \mathrm{d} V o l
$$

in which $E$ is the elastic modulus and $G$ is the shear modulus. For the beam-column representation, Eq. 10 becomes

$$
\begin{aligned}
U= & \frac{E A}{2} \int_{L}\left(\frac{\mathrm{d} u}{\mathrm{~d} x}\right)^{2} \mathrm{~d} x+\frac{P}{2} \int_{L}\left(\frac{\mathrm{d} v}{\mathrm{~d} x}\right)^{2} \mathrm{~d} x+\frac{P}{2} \int_{L}\left(\frac{\mathrm{d} w}{\mathrm{~d} x}\right)^{2} \mathrm{~d} x+\frac{E I_{z}}{2} \int_{L}\left(\frac{\mathrm{d}^{2} v}{\mathrm{~d} x^{2}}\right)^{2} \mathrm{~d} x \\
& +\frac{E I_{y}}{2} \int_{L}\left(\frac{\mathrm{d}^{2} w}{\mathrm{~d} x^{2}}\right)^{2} \mathrm{~d} x+\frac{G J}{2} \int_{L}\left(\frac{\mathrm{d} \phi}{\mathrm{d} x}\right)^{2} \mathrm{~d} x
\end{aligned}
$$

in which $E A$ is the axial rigidity, $E I_{y}$ and $E I_{z}$ are the flexural rigidities and $G J$ is the torsional rigidity.

The elastic stiffness relationship for a general fourth-order element is derived from the total potential energy $\Pi$ in terms of displacements; the total potential energy for nonlinear analysis being the sum of the internal strain energy $U$ in Eq. 11 and of the external work done $V$, which can be written as

$$
\begin{aligned}
\Pi= & \frac{E A}{2} \int_{L}\left(\frac{\mathrm{d} u}{\mathrm{~d} x}\right)^{2} \mathrm{~d} x+\frac{P}{2} \int_{L}\left(\frac{\mathrm{d} v}{\mathrm{~d} x}\right)^{2} \mathrm{~d} x+\frac{P}{2} \int_{L}\left(\frac{\mathrm{d} w}{\mathrm{~d} x}\right)^{2} \mathrm{~d} x+\frac{E I_{z}}{2} \int_{L}\left(\frac{\mathrm{d}^{2} v}{\mathrm{~d} x^{2}}\right)^{2} \mathrm{~d} x \\
& +\frac{E I_{y}}{2} \int_{L}\left(\frac{\mathrm{d}^{2} w}{\mathrm{~d} x^{2}}\right)^{2} \mathrm{~d} x+\frac{G J}{2} \int_{L}\left(\frac{\mathrm{d} \phi}{\mathrm{d} x}\right)^{2} \mathrm{~d} x-\mathbf{u}_{k}^{\mathrm{T}} \mathbf{f}_{k}
\end{aligned}
$$

where $\mathbf{u}_{k}$ and $\mathbf{f}_{k}$ are the column vectors of the incremental displacements and external applied forces with respect to the corresponding freedom, with $\mathbf{u}_{k}=\left\{\Delta u, \Delta \theta_{z 1}, \Delta \theta_{z 2}, \Delta \theta_{x}, \Delta \theta_{y 1}, \Delta \theta_{y 2}\right\}^{\mathrm{T}}$ in which $\Delta u$ $=u_{1}-u_{2}$ and $\Delta \theta_{x}=\phi_{1}-\phi_{2}$. Since the kinematic deformations in the present non-linear analysis rely on an updated Lagrangian description, the total potential energy equation which governs the problem can be linearised in each load increment, so that incremental displacements are utilised in each load increment of the total potential energy equation from which the incremental stiffness formulation for the proposed second-order analysis is derived.

The principle of virtual displacements is used in the present paper to derive the incremental secant $\left(\mathbf{K}_{s}\right)$ stiffness in the form of member resistance and tangent $\left(\mathbf{K}_{t}\right)$ stiffness matrices. Since the strain energy functional in Eq. 11 depends not only on the dependent variables $\mathbf{u}_{k}$, but also on the axial load parameter $q$, invoking Castigliano's first theorem of strain energy, as also given by Oran $[12,13]$ produces 
$\mathbf{K}_{s}=\frac{\partial U}{\partial \mathbf{u}_{k}}+\frac{\partial U}{\partial q} \cdot \frac{\partial q}{\partial \mathbf{u}_{k}}$

Using this, the secant stiffness formulation can be obtained from

$$
\begin{gathered}
M_{\alpha 1}=\frac{\partial U}{\partial \theta_{\alpha 1}}=\frac{E I_{\alpha}}{L}\left[\frac{9216+3456 q / 5+68 q^{2} / 5+8 q^{3} / 105}{(48+q)^{2}}\right] \Delta \theta_{\alpha 1} \\
+\frac{E I_{\alpha}}{L}\left[\frac{4608+576 q / 5+2 q^{2}+q^{3} / 42}{(48+q)^{2}}\right] \Delta \theta_{\alpha 2}
\end{gathered}
$$

or

$$
\begin{aligned}
M_{\alpha 1}= & \frac{E I_{\alpha}}{L}\left(C_{1} \Delta \theta_{\alpha 1}+C_{2} \Delta \theta_{\alpha 2}\right), \\
M_{\alpha 2}= & \frac{\partial U}{\partial \theta_{\alpha 2}}=\frac{E I_{\alpha}}{L}\left[\frac{9216+3456 q / 5+68 q^{2} / 5+q^{3} / 105}{(48+q)^{2}}\right] \Delta \theta_{\alpha 2} \\
+ & \frac{E I_{\alpha}}{L}\left[\frac{4608+576 q / 5+2 q^{2}+q^{3} / 42}{(48+q)^{2}}\right] \Delta \theta_{\alpha 1}
\end{aligned}
$$

or

$$
M_{\alpha 2}=\frac{E I_{\alpha}}{L}\left(C_{1} \Delta \theta_{\alpha 2}+C_{2} \Delta \theta_{\alpha 1}\right)
$$

in which the subscript $\alpha$ denotes $y$ or $z$, and

$$
\begin{aligned}
\Delta P= & P_{1}-P_{2}=\frac{\partial U}{\partial e}+\frac{\partial U}{\partial q} \cdot \frac{\partial q}{\partial e}=\frac{E A e}{L}+E A \sum_{\alpha=y, z}\left[\frac{16 q^{2} / 35}{(48+q)^{3}}\right]\left(\Delta \theta_{\alpha 1}-\Delta \theta_{\alpha 2}\right)^{2} \\
& =E A\left[\frac{e}{L}+\sum_{\alpha=y, z} C_{b}\right]
\end{aligned}
$$

where $e=\Delta u=u_{1}-u_{2}$ is the axial shortening with respect to the incremental axial member load $\Delta P$ as a dependent variable.

The tangent stiffness matrix can be obtained from the second derivative of the total potential functional in Eq. 12 with respect to the dependent variables $\mathbf{u}_{k}$ and the load parameter $q$, which includes the second order $\mathrm{P}-\delta$ effect. When the external work done $V$ is linear, the tangent stiffness can be written as the second derivative of $U$ with respect to $\mathbf{u}_{k}$ as

$$
\mathbf{K}_{t}=\frac{\partial^{2} \Pi}{\partial \mathbf{u}_{j} \partial \mathbf{u}_{k}}=\frac{\partial}{\partial \mathbf{u}_{j}}\left(\frac{\partial U}{\partial \mathbf{u}_{k}}\right)+\frac{\partial}{\partial \mathbf{u}_{k}}\left(\frac{\partial U}{\partial q} \cdot \frac{\partial q}{\mathbf{u}_{j}}\right) .
$$

The tangent stiffness for the fourth-order element, which relates the incremental deformation to the corresponding external loads imposed on the element in the member coordinate system, is then given as 


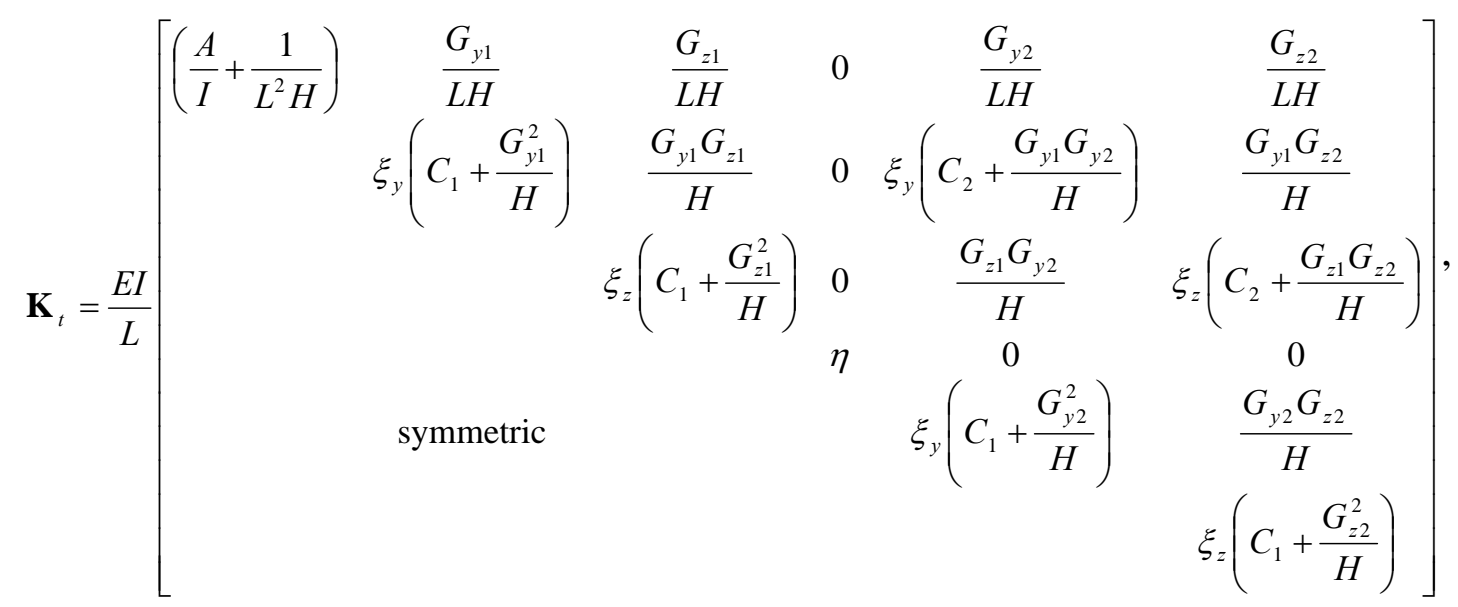

in which $I$ is the second moment of area about the relevant axis for which buckling is to be considered, $\xi_{y}=I_{y} / I, \xi_{z}=I_{z} / I$ and the coefficients $G_{\alpha i}$ and $H$ are given in the Appendix. When the axial force parameter $q$ is taken as zero, the stiffness terms $G_{\alpha i}$ vanish and the tangent stiffness matrix in Eq. 20 is the same as that for a conventional cubic element.

For the global coordinate system, the tangent stiffness $\mathbf{K}_{T}$ is represented as

$$
\mathbf{K}_{T}=\sum_{\text {elements }} \mathbf{L} \mathbf{K}_{e} \mathbf{L}^{\mathrm{T}}=\sum_{\text {elements }} \mathbf{L}\left(\mathbf{T}^{\mathrm{T}} \mathbf{K}_{t} \mathbf{T}+\mathbf{N}\right) \mathbf{L}^{\mathrm{T}},
$$

where $\mathbf{T}$ is a transformation matrix which relates the member forces to the element forces in the local coordinate system, $\mathbf{L}$ is a transformation of the local coordinates to global coordinates, and $\mathbf{N}$ is a stability matrix to allow for the work done by rigid body motions or changes of the geometry of the structure. Consequently, the geometry of the structure is then updated by accumulating both the 'neutral' member deformations and rigid body movements in an updated Lagrangian approach. Because the governing equation for total potential energy in Eq. 12 on which the stiffness matrices have been obtained is non-linear, an incremental-iterative procedure is needed to trace the non-linear equilibrium path.

\section{NUMERICAL VERIFICATIONS}

The proposed non-linear analysis formulation of this paper which handles geometric non-linearity is investigated in this section for a cantilever undergoing a large tip deflection, as well as for three frame structures. A two-member right angled frame is investigated, as well as a two-bar toggle frame and a two-bar truss, and finally a 24-member reticulated shallow dome structure is investigated, in which snap-through buckling, post-buckling and large displacement can occur.

\subsection{Large Displacement of a Cantilever}

The large displacement analysis of an elastic cantilever beam was first presented by Bisshopp and Drucker [14], in which an exact theoretical solution was developed. Their analysis allows for expressions of the exact curvature and the effect of shortening due to the deflection of the tip load on the cantilever. This problem was also investigated by Saleeb and Chen [15], and others. For the modelling of this paper, four elements were used for the cantilever. 


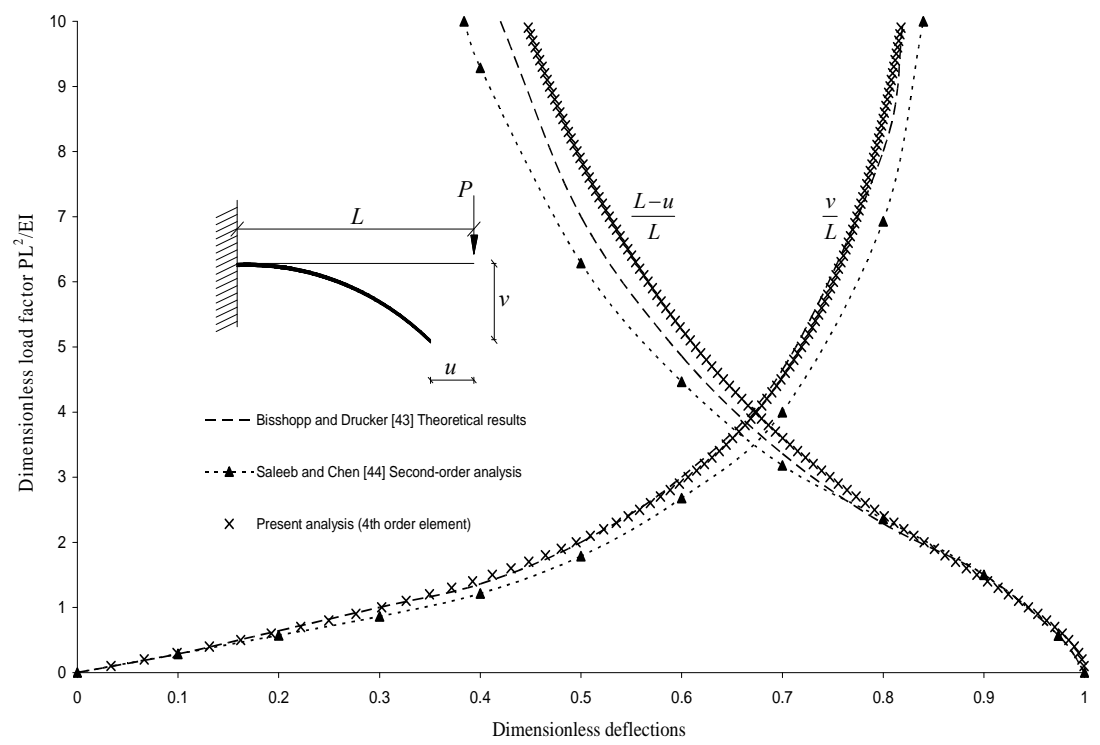

Figure 3. Large Displacement Analysis of Tip-loaded Cantilever

Figure 3 shows the normalised horizontal and vertical deflections of the free end, plotted against the dimensionless applied load $P L^{2} / E I$. It can be seen that the axial deformations $u$ from the present analysis are in good agreement with the theoretical solution [14], with the transverse deformations $v$ coinciding with the theoretical solution throughout the entire range. The proposed non-linear modelling therefore allows large displacement behaviour due to bending and shortening to be simulated.

\subsection{Load-deflection Response of Right Angled Frame}

Koiter [16] provided the first analytical solution of a right angled frame, presenting a formulation for studying its buckling and post-buckling response. Chan and Zhou [4] have also studied this frame in order to verify their higher-order element formulation. A right-angled frame with pin supports and equal column and beam lengths, subjected to a point load $P$ with an eccentricity $e$ $(0.254 \mathrm{~m})$ to the beam-to-column joint, was modelled in the present analysis with two elements for each member. The section, geometry and material properties are shown in Figure 4, which also shows the joint rotation plotted against the dimensionless load $P / P_{E}$, where $P_{E}$ is the Euler load. The proposed non-linear modelling produces results consistent with those of Chan and Zhou [4], and moreover predicts the post-buckling response of a perfect frame given by Koiter [16].

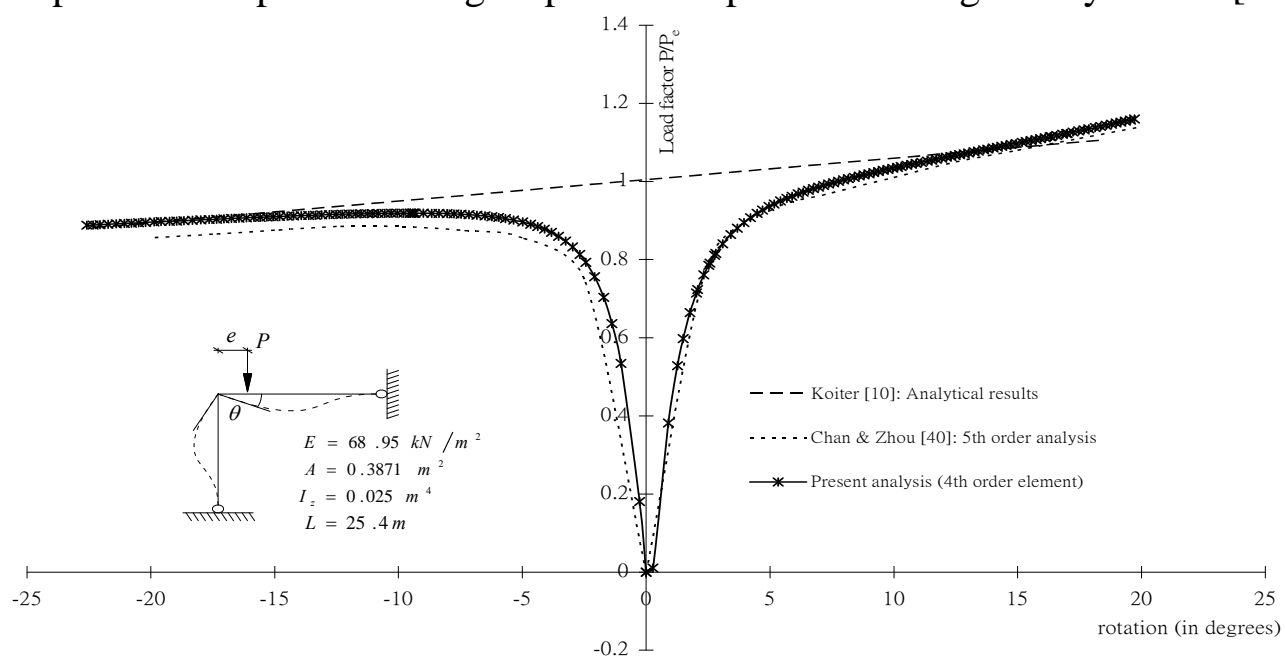

Figure 4. Large Displacement of Right-angle Frame 


\subsection{Snap-through Buckling and Post-buckling of Toggle Frame}

A shallow two-bar frame (toggle frame) was proposed by Williams [17] for demonstrating the snap-through buckling behaviour of a structure, and he presented experimental and analytical solutions for a toggle frame with fixed end supports under a vertical load $P$ at its apex. The elastic modulus of the bars is $71.02 \mathrm{kN} / \mathrm{mm}^{2}$ and they are of rectangular section with a width of 19.126 $\mathrm{mm}$ and depth of $6.1722 \mathrm{~mm}$. For the modelling of this paper, four elements were used for each bar.

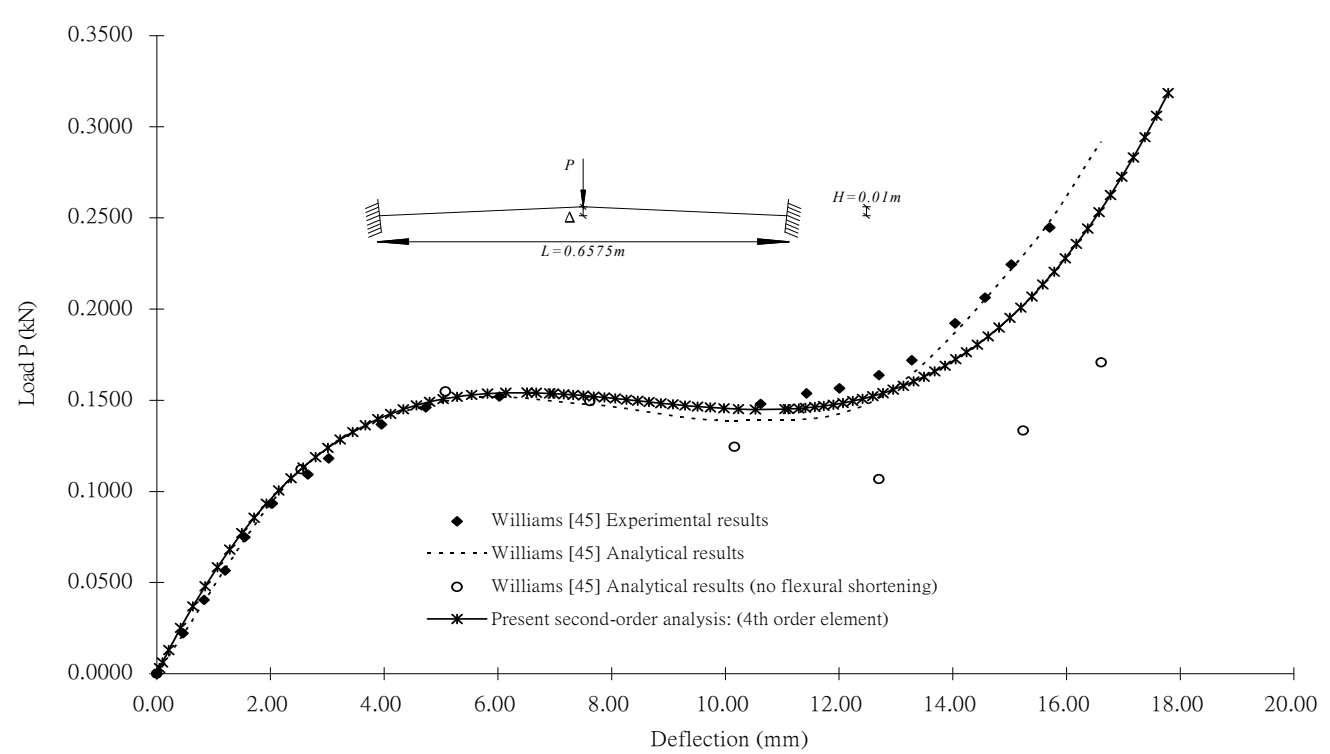

Figure 5. Load-deflection curve and snap-through buckling of toggle frame

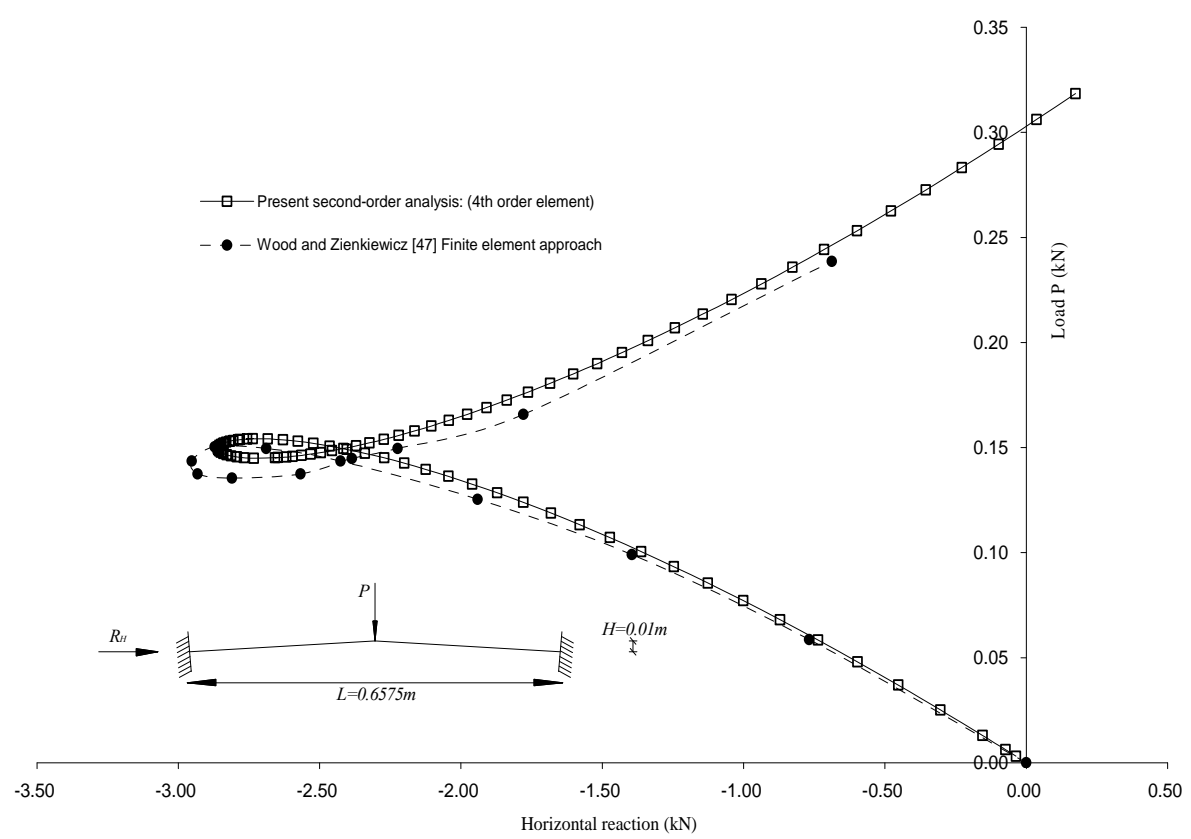

Figure 6. Horizontal reaction and snap-through buckling of toggle frame

Figure 5 shows the toggle frame with a rise of $100 \mathrm{~mm}$ and a horizontal span of $657.5 \mathrm{~mm}$ between supports, for which the vertical deflection at the apex is shown as a function of the load. After attaining the critical load for the frame, the structure softens slightly with increasing deformations as the load decreases, and then hardens as the frame is subjected to tension; similar responses occur with very shallow arches with fixed supports [18]. It can be seen from the figure that the experimental results are disparate from the analytical ones when they do not include flexural shortening, but the experiments agree well with the results of the present paper which include 
flexural shortening, as well as those of Williams [17] when flexural shortening is included. Wood and Zienkiewicz [19] analysed Williams' toggle frame using the finite element method with a modified Newton-Raphson technique, and presented results for the horizontal reaction $R_{H}$ shown in Figure 6; their result for the load-deflection curve was consistent with that of Williams [17]. Because of the flatness of the toggle frame, these reactions are very similar to the forces in the two members. The results from the present formulation are also shown in Figure 6, and are in good agreement with those of Wood and Zienkiewicz [19]. During the snap-through bucking of the toggle frame, which is much beyond Euler load of $2.4 \mathrm{kN}$, the member force decreases slightly, and the compression gradually reduces with an increase of the applied load until $R_{H}$ becomes positive and the members are in tension, as shown in the Figure 6.

\subsection{Snap-through Buckling and Post-buckling of Two-member Plane Truss}

A two-member truss is used as a demonstration of the snap-through buckling and post-buckling of a plane truss which is subjected to a vertical load $P$ at its vertex. This plane truss has a rise of 25.4 $\mathrm{mm}$ and clear span of $1.27 \mathrm{~m}$ between two pinned supports, as shown in Figure 7 . Each member of the truss is assumed to have the same cross-sectional area $A$ and elastic modulus $E$. For the modelling of this paper, four elements were used for each member of this truss.

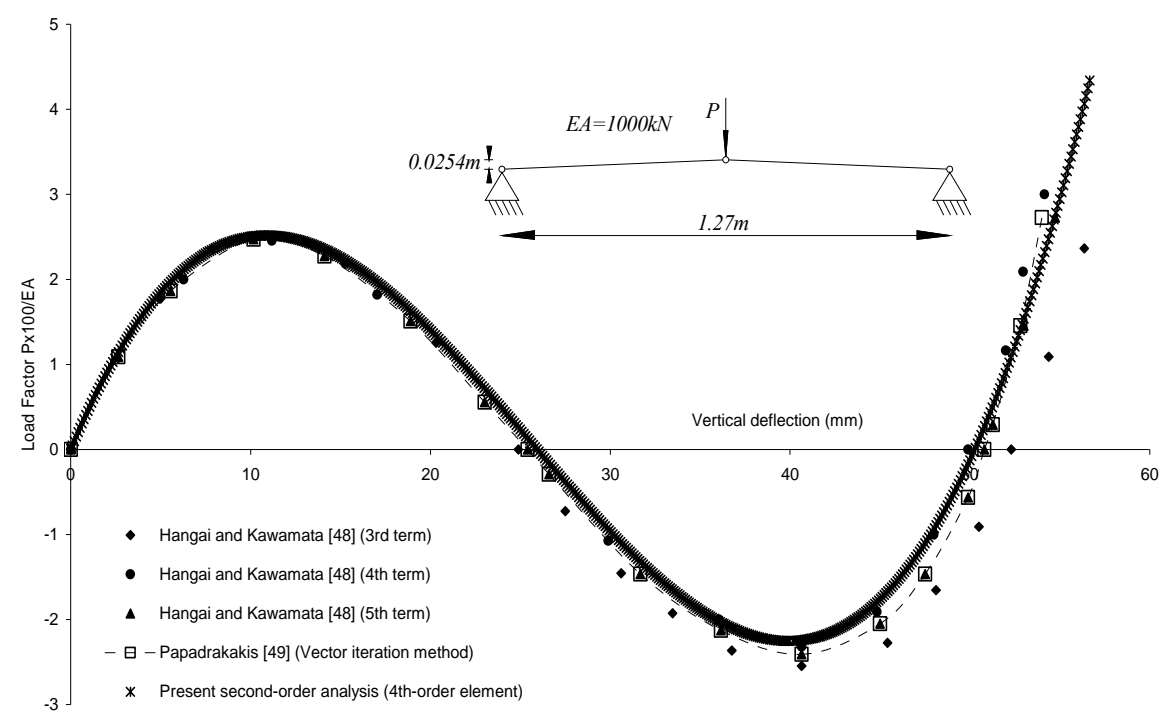

Figure 7. Load-deflection Curves of the Plane Truss

Hangai and Kawamata [20] previously analysed this truss by the static perturbation technique, using various degrees of approximation in the force-displacement relationship. For the same plane truss, Papadrakakis [21] presented a vector iteration method to trace the non-linear load-deflection relationship, including snap-through and post-bucking responses. Figure 7 plots the vertical deflection at vertex against dimensionless load factor $P /(E A)\left(\times 10^{2}\right)$ from the present analysis as well as the former methods $[20,21]$. It was found that the present load-deflection curve is in good agreement with the results $[20,21]$ over the entire loading regime, except that using the third-term approximation from Hanagai and Kawamata [20]. In particular, the post-buckling behaviour from this approximation is disparate from the others. The proposed non-linear analysis therefore adequately accounts for snap-through buckling and post-buckling of the truss structures. 


\subsection{Shallow Reticulated Shell Structure}

A 24-member reticulated shallow shell which was studied by Hangai and Kawamata [20] has been analysed by the proposed method of this paper. All members are identical geometrically and materially, and all joints and supports are pinned (the supports are fixed in direction, being unable to transmit bending moments or torques). For this study, a point load is applied at the top of the reticulated shell at node 1, as shown in Figure 8 which also shows the geometry of the shell structure. This reticulated shell experiences snap-through and post-buckling characteristics, as well as large displacements and rotations at its joints (as reported in [20]), and so is an ideal benchmark problem for large-displacement second-order analysis. The reticulated shell structure was also studied by Jagannathan et al. [22], Holzer et al. [23] and Papadrakais [21].
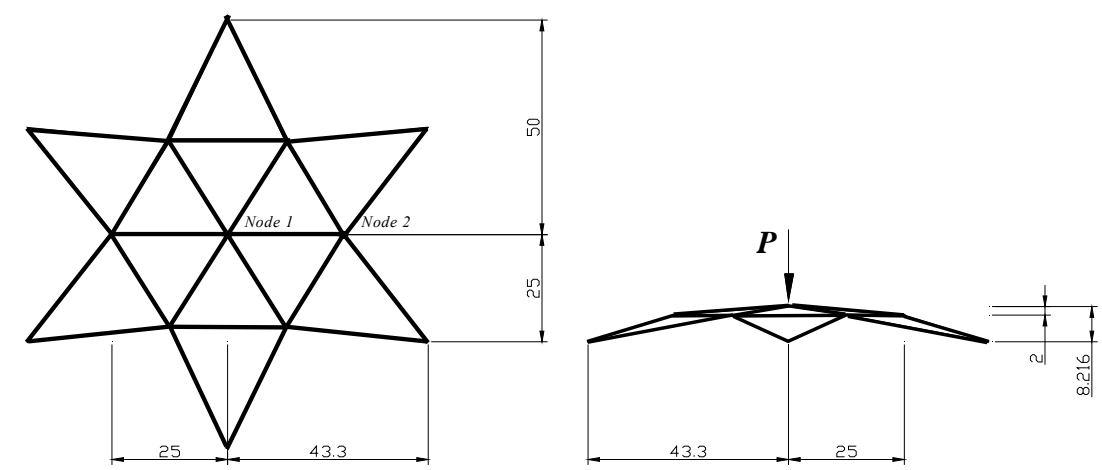

Figure 8. Geometry of 24-member Reticulated Shell Structure

In the analysis of this shell using the proposed formulation, one element was used to model each member of the reticulated structure, and Figure 9 shows the vertical displacement of node 1, plotted against the dimensionless load $P /(E A)\left(\times 10^{4}\right)$, and it can be seen that the results are consistent with those of $[22,23]$ throughout the loading and unloading portions of the curve. Figures 10 and 11 show the vertical and horizontal displacements of node 2 (in Figure 8) respectively, from which it can be seen that the present method agrees very well with the results of Holzer [23], but is disparate with that of Hangai and Kawamata [20] and well as of Jagannathan et al. [22]. It appears from Figs. 9 to 11 that Hangai and Kawamata's solution is not in agreement with those of other investigations, including that proposed in this paper, and this is attributable to the large member rotations at the nodes in which the rigid body rotations also contribute to the strain energy in their formulation. It is interesting to note that there are two zero-load configurations for this shell; at the first the vertical deformations of nodes 1 and 2 are approximately $19 \mathrm{~mm}$ and $-1.16 \mathrm{~mm}$ respectively, at the second they are $40 \mathrm{~mm}$ and $0 \mathrm{~mm}$. Because node 1 is located $20 \mathrm{~mm}$ above the horizontal plane containing the internal ring nodes before loading and $20 \mathrm{~mm}$ below it in the second zero-load configuration, the latter state is symmetrical to the initial unloaded state with respect to this plane and the horizontal deflections are zero, as can be seen from Figs. 10 and 11. The present study therefore allows for large strains, with the large rotations of the shell being captured accurately. 


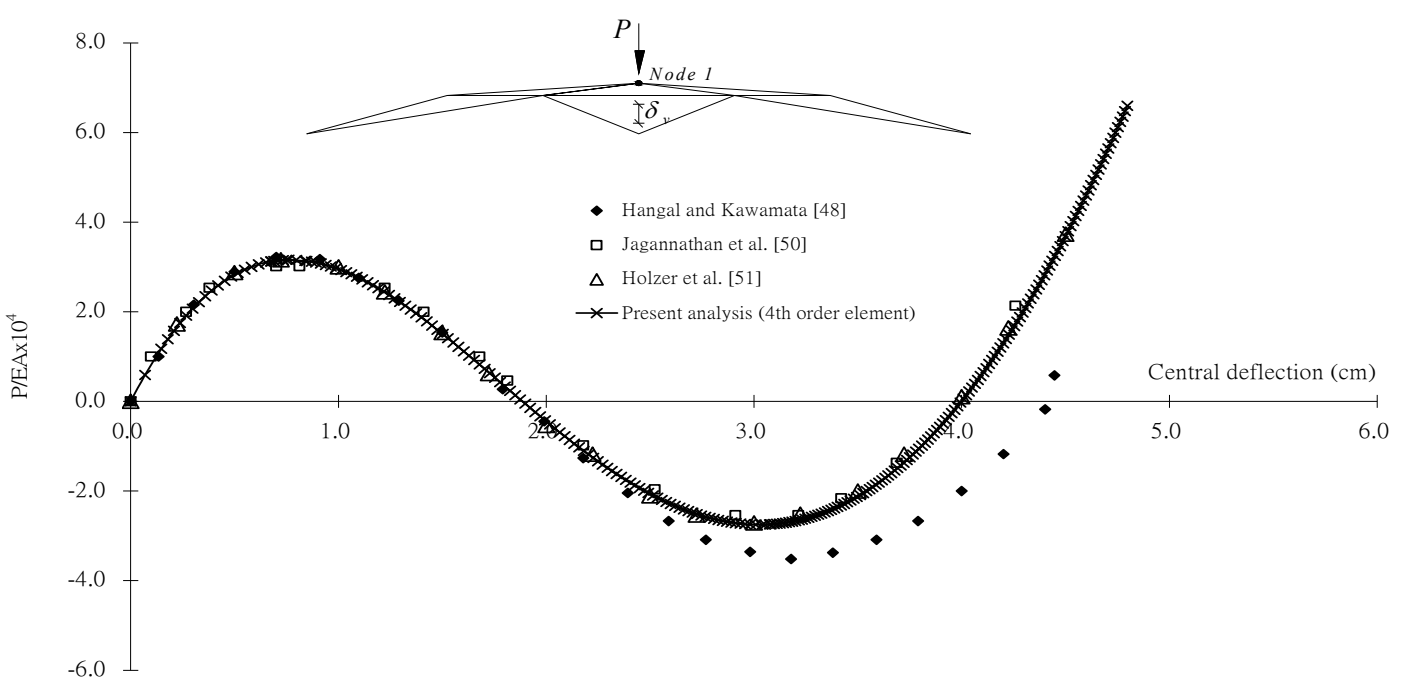

Figure 8. Load vs central deflection displacement for node 1 of reticulated shell

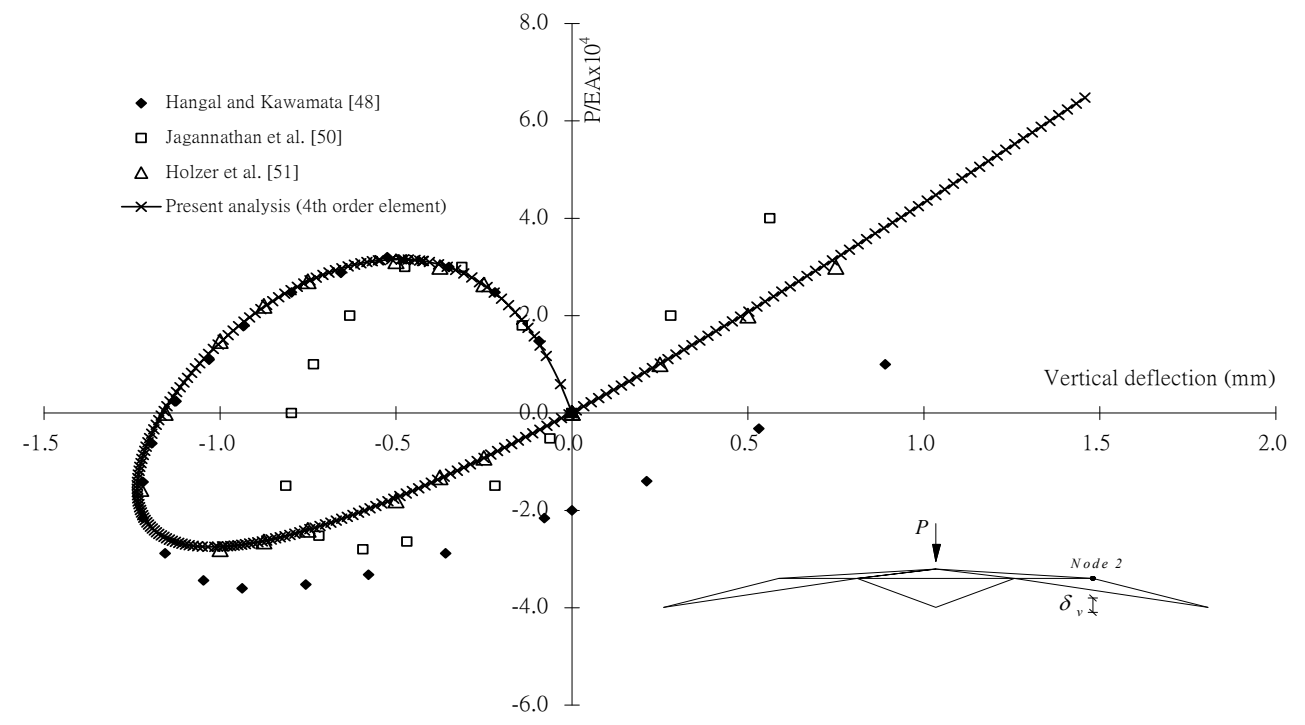

Figure 9. Load vs central deflection displacement for node 2 of reticulated shell

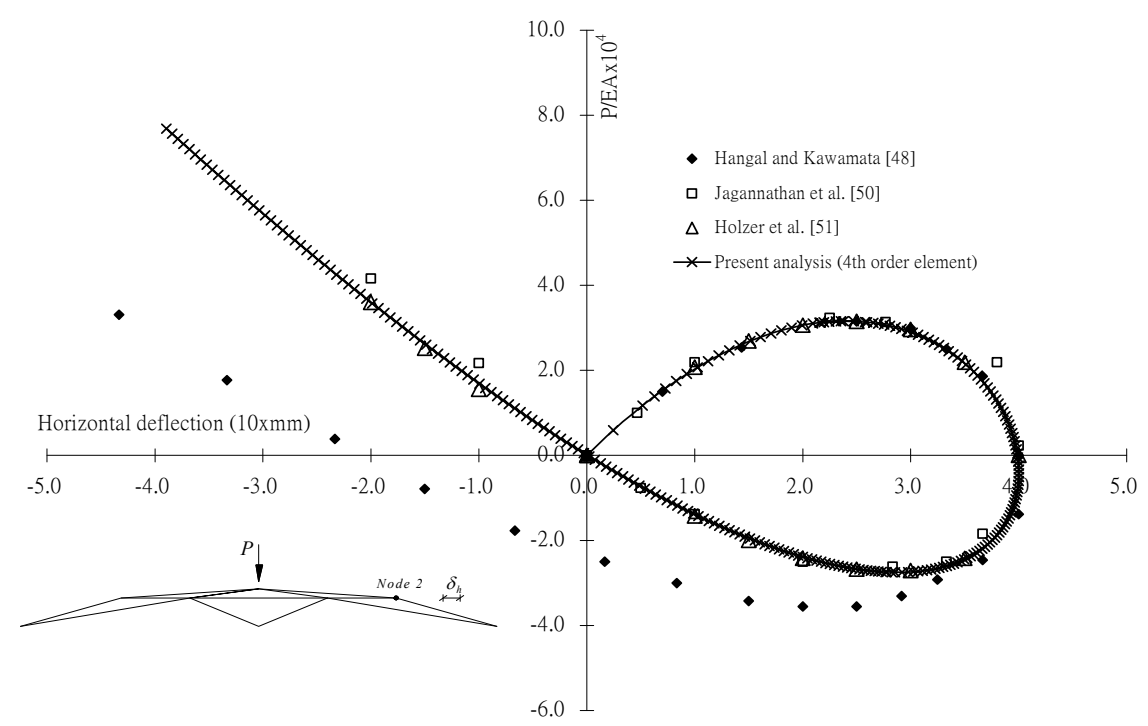

Figure 10. Load vs horizontal deflection displacement for node 2 of reticulated shell 


\section{CONCLUDING REMARKS}

This paper has presented a non-linear finite element formulation using a higher-order element to fulfil a need for versatile, reliable and efficient non-linear analysis of general steel framed structures with very many members. A numerical analysis of this type is a useful formulation for evolution of a method for determining structural responses which occur under extreme loading scenarios such as those of fire and blast, and for which a plethora of scenarios must be considered in design and assessment. The fourth-order element, derived by fulfilling the kinematic conditions needed for the finite element technique and implemented in an incremental updated Lagrangian description, has been shown to predict flexural buckling, snap-through buckling and large displacement post-buckling behaviour of typical structures whose responses have been reported by independent researchers. The technique further forms a basis for the refined plastic hinge approach of the companion paper for handling material non-linearity. In some cases, use of a single element for a member may not be appropriate to capture correctly the geometric non-linearity associated with flexural buckling and snap-through buckling which is attributable to the proposed asymmetric and incomplete bowing function of this paper. Hence at least two elements per member are suggested for these isolated cases. In particular, it was observed from the numerical verifications that four elements per member can, in general, replicate accurate and efficient solutions for structures encountering geometric non-linearities.

\section{ACKNOWLEDGEMENT}

The work in this paper was supported by the Australian Research Council through a Federation Fellowship awarded to the second author.

\section{REFERENCES}

[1] Wen, R.K. and Rahimzadeh, J., "Nonlinear Elastic Frame Analysis by Finite Elements", Journal of Structural Engineering, ASCE, 1983, Vol. 109, No. 8, pp. 1952-1971.

[2] Meek, J.L. and Tan, H.S., "Geometrically Nonlinear Analysis of Space Frames by An Incremental Iterative Technique", Computer Methods in Applied Mechanics and Engineering; 1984, Vol. 47, pp. 261-281.

[3] So, A.K.W. and Chan, S.L., "Buckling and Geometrically Nonlinear Analysis of Frames Using One Element/Member”, Journal of Constructional Steel Research, 1991, Vol. 20, pp. 271-289.

[4] Chan, S.L. and Zhou, Z.H., "Pointwise Equilibrating Polynomial Element for Nonlinear Analysis of Frames”, Journal of Structural Engineering, ASCE, 1994, Vol. 120, No. 6, pp. 1703-1717.

[5] Chan, S.L. and Zhou, Z.H., "Second-order Elastic Analysis of Frames Using Single Imperfect Element Per Member”, Journal of Structural Engineering, ASCE, 1995, Vol. 121, No. 6, pp. 939-945.

[6] Gu, J.X. and Chan, S.L., "A Refined Finite Element Formulation for Flexural and Torsional Buckling of Beam-column with Finite Rotations”, Engineering Structures, 2005, Vol. 27, pp. 749-759.

[7] Liew, J.Y.R., Chen, H., Shanmugam, N.E. and Chen, W.F., "Improved Nonlinear Plastic Analysis of Space Frame Structures”, Engineering Structures, 2000, Vol. 22, pp. 1324-1338.

[8] Liew, J.Y.R., Tang, L.K. and Choo, Y.S., "Advanced Analysis for Performance-based Design of Steel Structures Exposed to Fires”, Journal of Structural Engineering, ASCE, 2002, Vol. 128, No. 12, pp. 1584-1593. 
[9] Liew, J.Y.R. and Chen, H., "Exposion and Fire Analysis of Steel Frames Using Fiber Element Approach”, 2004, Journal of Structural Engineering, ASCE, Vol. 130, No. 7, pp. 991-1000.

[10] Iu, C.K. and Chan, S.L., "A Simulation-based Large Deflection and Inelastic Analysis of Steel Frames Under Fire”, Journal of Constructional Steel Research, 2004, Vol. 60, pp. 1495-1524.

[11] Iu, C.K., Chan, S.L. and Zha, X.X., "Nonlinear Pre-fire and Post-fire Analysis of Steel Frames”, Engineering Structures, 2005, Vol. 27, pp. 1689-1702.

[12] Oran, C., "Tangent Stiffness in Plane Frames”, Journal of the Structural Division, ASCE, 1973, Vol. 99, No. ST6, pp. 973-985.

[13] Oran, C., "Tangent Stiffness in Space Frames”, Journal of the Structural Division, ASCE, 1973, Vol. 99, No. ST6, pp. 987-1001.

[14] Bisshopp, K.E. and Drucker, D.C., "Large Deflection of Cantilever Beams”, Quarterly Journal of Applied Mathematics, 1945, Vol. 3, No. 3, pp. 272-275.

[15] Saleeb, A.F. and Chen, W.F., "Elastic-plastic Large Displacement Analysis of Pipes", Journal of Structural Engineering, ASCE, 1981, Vol. 107, No. 4, pp. 605-626.

[16] Koiter, W.T., "Post-buckling Analysis of a Simple Two-bar Frame", Recent Progress in Applied Mechanics (Broberg et al. eds), New York: John Wiley and Sons, 1967, pp. 337-354.

[17] Williams, F.W., "An Approach to the Non-linear Behaviour of Members of a Rigid Jointed Plane Framework with Finite Deflections”, Quarterly Journal of Mechanics and Applied Mathematics, 1964, Vol. 17, No. 4, pp. 451-469.

[18] Bradford, M.A. and Uy, B. and Pi, Y-L., "In-plane Elastic Stability of Arches under a Central Concentrated Load”, Journal of Engineering Mechanics, ASCE, 2002, Vol. 128, No. 7, pp. 710-719.

[19] Wood, R.D. and Zienkiewicz, O.C., "Geometrically Nonlinear Finite Element Analysis of Beams, Frames, Arches and Axisymmetric Shells”, Computers and Structures, 1977, Vol. 4, pp. 725-735.

[20] Hangai, Y. and Kawamata, S., "Nonlinear Analysis of Space Frames and Snap-through Buckling of Reticulated Shell Structures”, Proceedings International Association for Shell and Spatial Structures (IASS) Pacific Symposium, Tokyo, 1971, pp. 803-816.

[21] Papadrakais, M., "Post-buckling Analysis of Spatial Structures by Vector Iteration Methods”, Computers and Structures, 1981, Vol. 14, No. 5-6, pp. 393-402.

[22] Jagannathan, D.S., Epstein, H.I. and Christiano, P.P., "Snap-through Buckling of Reticulated Shells”, Proceedings of the Institution of Civil Engineers London, 1975, Part 2, Vol. 59, pp. 727-742.

[23] Holzer, S.M., Plaut, R.H. and Somer, A.E., "Stability of Lattice Structures under Combined Loads”, Journal of the Engineering Mechanics Division, ASCE, 1980, Vol. 106(EM2), pp. 289-305. 


\section{APPENDIX}

The terms $G_{\alpha i}(\alpha=y$ or $z, i=1$ or 2$)$ in Eq. 25 are:

$$
\begin{gathered}
\frac{\partial M_{\alpha 1}}{\partial q}=\frac{\partial}{\partial \theta_{\alpha 1}}\left(\frac{\partial U}{\partial q}\right)=\frac{E I}{L}\left[\frac{32 q^{2} / 35}{(48+q)^{3}}\right]\left(\Delta \theta_{\alpha 1}-\Delta \theta_{\alpha 2}\right)=\left(\frac{E I}{L}\right) G_{\alpha 1} \\
\frac{\partial M_{\alpha 2}}{\partial q}=\frac{\partial}{\partial \theta_{\alpha 2}}\left(\frac{\partial U}{\partial q}\right)=\frac{E I}{L}\left[\frac{-32 q^{2} / 35}{(48+q)^{3}}\right]\left(\Delta \theta_{\alpha 1}-\Delta \theta_{\alpha 2}\right)=\left(\frac{E I}{L}\right) G_{\alpha 2} \\
\frac{\partial q}{\partial \theta_{\alpha 1}}=\frac{\frac{I}{A L^{2}}-\sum_{\alpha=y, z}\left[\frac{1536 q / 35-16 q^{2} / 35}{(48+q)^{4}}\right]\left(\Delta \theta_{\alpha 1}-\Delta \theta_{\alpha 2}\right)^{2}}{H}=\frac{G_{\alpha 1}}{H} \\
\frac{\partial q}{\partial \theta_{\alpha 2}}=\frac{\left[\frac{-32 q^{2} / 35}{(48+q)^{3}}\right]\left(\Delta \theta_{\alpha 1}-\Delta \theta_{\alpha 2}\right)}{\frac{I}{A L^{2}}-\sum_{\alpha=y, z}\left[\frac{1536 q / 35-16 q^{2} / 35}{(48+q)^{4}}\right]\left(\Delta \theta_{\alpha 1}-\Delta \theta_{\alpha 2}\right)^{2}}=\frac{G_{\alpha 2}}{H} \\
\frac{\partial q}{\partial e}=\frac{1 / L}{\frac{I}{A L^{2}}-\sum_{\alpha=y, z}\left[\frac{1536 q / 35-16 q^{2} / 35}{(48+q)^{4}}\right]\left(\Delta \theta_{\alpha 1}-\Delta \theta_{\alpha 2}\right)^{2}}=\frac{1}{H L}
\end{gathered}
$$

